\title{
The influence of the environment on the jet of an AGN
}

Núria Torres-Albà* and Valentí Bosch-Ramon

Departament de Física Quàntica i Astrofísica, Institut de Ciències del Cosmos (ICC), Universitat de Barcelona (IEEC-UB), Martí i Franquès 1, E08028 Barcelona, Spain

E-mail: ntorres@fqa.ub.edu

AGN jets have an impact on the galaxy that hosts them, and in turn the galaxy can have an influence on the jet itself. In this work, we give a brief overview of previous studies regarding the interaction of an AGN jet and its environment. We then focus on recent results regarding the possibility of stars introducing stellar-wind and ISM material into the jet at the moment of penetration, and on whether this phenomenon can have a significant impact on the jet, be it dynamically or through emission of non-thermal radiation.

Frontier Research in Astrophysics - III (FRAPWS2018)

28 May - 2 June 2018

Mondello (Palermo), Italy

${ }^{*}$ Speaker. 


\section{Introduction}

Galaxies host supermassive black holes in their innermost regions, which may start actively accreting nearby material, thus becoming an Active Galactic Nucleus (AGN). The process of accretion, together with the presence of strong magnetic fields, can trigger the launching of bipolar, relativistic outflows, or jets (e.g. [1]). In these jets, particles are accelerated up to very high energies, generally resulting in non-thermal emission, particularly in radio wavelengths and gamma-rays. For a general review in the topic of jet formation we refer the reader to e.g. [2, 3, 4].

These jets can have heights far larger than the galaxy itself, and through their propagation they inevitably interact with a variety of objects present within the host. Particularly, the inner regions of the galaxy are rich in gas, clouds and stars, which may penetrate the jet. Previous works are numerous, and include studies of the interaction between the jet and broad-line region clouds (e.g. $[5,6,7]$ ), narrow-line region clouds (e.g. [8]), stars (e.g. $[9,10,11,12,13,14,15,16]$ ), and even whole globular clusters (e.g. [17]).

In this work we review previous results, as well as give a description of the interaction between the jet and any object that may be present within the galaxy. We also provide quantitative estimates of how a population of red giants can interact with the jet of a massive elliptical galaxy as the stars penetrate it. Finally, a discussion is provided.

\section{Obstacles within the jet}

Obstacles (i.e. clouds, stars, clusters) that penetrate the jet can mainly result in a dynamical impact in the jet and/or in the generation of non-thermal emission.

\subsection{Jet Deceleration and Disruption}

Jets can be decelerated mainly through exchange of momentum with external matter, which manages to penetrate into the jet. Radio observations show extragalactic jets with morphologies that suggest deceleration: large opening angles, dimmer edges and lower velocities (Fanaroff \& Riley I, FR I, [18]).

As jets propagate through a gas-rich environment, entrainment occurs through mixing in the turbulent shear-layer. In astrophysical jets, turbulence is expected in boundary layers due to the very large Reynold numbers, and later grows both into the jet and into the environment, causing the initially laminar flow to become turbulent. As shown by numerical estimations and simulations, this process can efficiently decelerate a jet (e.g. [19, 20, 21]).

Numerical simulations also result in the possibility of decelerating the jet significantly through recollimation shocks. The initially overpressured jet expands through the Inter-Stellar Medium (ISM) until it becomes underpressured with respect to it, and then recollimates. Oscillations around pressure equilibrium and the generation of standing shocks lead to mass-loading through entrainment (see e.g. [22]).

Another possible mechanism of significant jet mass-loading is the interaction with a stellar wind. Young massive stars or red giants continuously lose large quantities of mass through their winds, and if present within the jet in large enough numbers, they can load it enough to result in a dynamical impact (e.g. $[23,24,12,16])$. 


\subsection{Gamma-ray emission}

Interactions between a cloud or star and the jet may result in acceleration of particles up to relativistic speeds, either through strong shocks or other mechanisms. The accelerated particles then radiate through Synchrotron emission in presence of strong magnetic fields, or inverse Compton (IC) in presence of strong radiation fields.

Focusing on the particular case of stars, particle acceleration occurs in a double bow-shock formed around the point of equilibrium between jet and stellar-wind ram pressures, the stagnation point, $R_{\mathrm{S}}$. For stars with strong winds, the interaction may be sufficient to produce detectable non-thermal radiation.

$$
P_{\mathrm{w}}=\rho v_{\mathrm{w}}^{2}=\frac{\dot{M} v_{\mathrm{w}}}{4 \pi R_{\mathrm{s}}^{2}}, \quad P_{\mathrm{j}} \simeq \frac{L_{\mathrm{j}}}{c S_{\mathrm{j}}}, \quad R_{\mathrm{s}}^{2}=\frac{c S_{\mathrm{j}} \dot{M} v_{\mathrm{w}}}{4 \pi L_{\mathrm{j}}}
$$

where $P_{\mathrm{w}}$ and $v_{\mathrm{w}}$ are the star wind ram pressure and velocity respectively and $\dot{M}$ its mass-loss rate. $P_{\mathrm{j}}, L_{\mathrm{j}}$ and $S_{\mathrm{j}}$ are the jet ram pressure, luminosity and section at a certain height, and $c$ the speed of light.

Previous works have studied stars with heavy mass-loss rates interacting near the base of the jet to explain possible gamma-ray outbursts (e.g. [25, 11]), or the interaction of whole stellar populations with the jet to explain persistent emission (e.g. $[13,17,16])$.

\section{Stellar Wind Bubbles}

In this work, we focus on a different means of stars introducing material into the jet: bubbles formed by the stellar wind as it accumulates around the star when it moves through the ISM.

When a star moves through the ISM, the stellar wind is in equilibrium with all external pressures present around the star (i.e. ISM pressure, $P_{\mathrm{ISM}}$, orbital pressure generated by its own movement, $P_{\text {orb }}$, jet lateral pressure when approaching the jet, $\left.P_{\text {lat }}\right)$.

$$
P_{\text {ext }}=\max \left(P_{\text {lat }}, P_{\mathrm{ISM}}+P_{\text {orb }}\right), \quad P_{\text {lat }}(z)=L_{\mathrm{j}} / c R_{\mathrm{j}}^{2}(z) \Gamma_{\mathrm{j}}^{2}, \quad P_{\text {orb }}(z)=\rho_{\mathrm{ISM}} v_{\text {orb }}(z)^{2},
$$

where $\Gamma_{\mathrm{j}}$ is the jet Lorentz factor and $\rho_{\mathrm{ISM}}$ the density of the ISM.

This pressure equilibrium determines the radius $R_{\mathrm{b}}$, and therefore mass $M_{\mathrm{b}}$, of the bubble of stellar material generated around the star. As the star penetrates the jet, it is impacted by the jet ram pressure, and eventually reaches pressure equilibrium with it. When that happens, the bubble surrounding the star has the size of the stagnation radius. The material contained between these two radii, $R_{\mathrm{b}}-R_{\mathrm{S}}$, can be lost within the jet in the form of a blob.

This blob is then impacted by the jet ram pressure and evolves upstreams, heating up and expanding as well as accelerating as described in $[10,26]$. In this process, part of the energy of the shock can be invested into the acceleration of non-thermal particles.

\subsection{Stellar Population and Jet Properties}

We focus on a population of red giants present in the bulge of an elliptical galaxy. We model this population exactly as in [16], taking as reference values those of the well-studied galaxy M87. 
This population of stars moves within the galaxy with a certain orbital velocity, and penetrates the jet at a rate [25]:

$$
\mathrm{d} P\left(z, t_{\mathrm{RG}}\right) \simeq n_{\mathrm{s}}\left(z, t_{\mathrm{RG}}\right) v_{\mathrm{orb}}(z) R_{\mathrm{j}}(z) \mathrm{d} z
$$

where $n_{\mathrm{s}}$ is the stellar number density.

We assume the jet is conical, with a constant half-opening angle that we fix at $\theta=0.1$, luminosity $L_{\mathrm{j}}=10^{44} \mathrm{erg} \mathrm{s}^{-1}$ and Lorentz factor $\Gamma=10$. We also take into account that the jet may recollimate at a height in which its pressure is equal to that of the ISM, which we fix at $P_{\mathrm{ISM}}=10^{-12} \mathrm{erg} \mathrm{cm}^{-2}$. We also assume the jet points toward the observer, in order to maximize Doppler boosting.

\subsection{Mass Loading}

The jet is loaded with external matter both through the mass-loss rates of stars constantly present within the jet (which we call steady-state interaction, see [16]) and the bubbles expelled by stars at penetration. The total mass loaded within the jet by the considered population is plotted in Fig. 1.

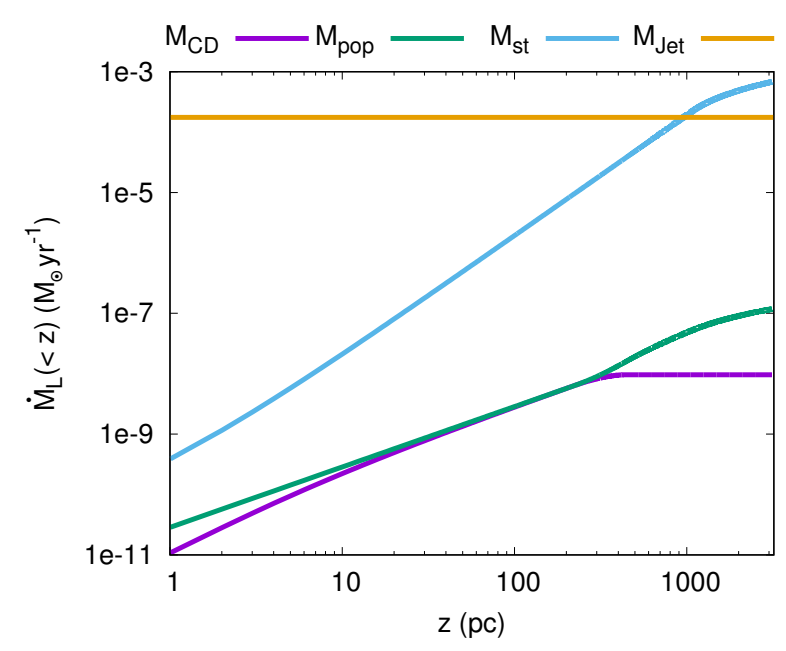

Figure 1: Total mass loaded into the jet at height $\mathrm{z}$ by the population of red giants: through wind-bubbles, into the contact discontinuity $\left(M_{\mathrm{CD}}\right)$ and the jet $\left(M_{\mathrm{pop}}\right)$, and through steady-state interaction $\left(M_{\mathrm{st}}\right)$. The loaded mass is compared to the jet's own mass-rate $\left(M_{\mathrm{Jet}}\right)$.

The mass loaded in the form of bubbles as stars penetrate the jet, both into the shear layer and within the jet, is compared to the jet $\dot{M}_{\mathrm{jet}}=L_{\mathrm{j}} / \Gamma_{\mathrm{j}} c^{2}$ and the mass loaded through stellar winds during the jet-crossing time of the stars. The latter is comparable to the mass-rate of the jet on scales of hundreds of parsec, meaning the number of stars permanently within the jet might be sufficient to affect it dynamically, as was seen by [16]. However, the mass loaded in the form of bubbles is various orders of magnitude lower, and therefore unlikely to result in a dynamical effect on the jet. 


\subsection{Non-Thermal Emission}

As the jet impacts the blob, it's accelerated in a characteristic time

$$
t_{\mathrm{acc}} \simeq\left\{\begin{array}{ll}
z_{0} / \beta c, & \text { if } D<1 \\
z_{0} / D \beta c, & \text { if } D>1
\end{array},\right.
$$

where $D$ is a dimensionless parameter related to jet energy and the mass of the bubble:

$$
D \equiv \frac{P_{0} \pi R_{\mathrm{b}}^{2} z_{0}}{4 c^{2} M_{\mathrm{b}} \Gamma_{\mathrm{j}}^{3}},
$$

(see e.g. [26]) where any subindex " 0 " refers to the value at $z_{0}$, height at which the star penetrates.

If this acceleration time is shorter than the characteristic time of energy losses, $t_{\text {acc }}^{\prime} \ll t_{\text {loss }}^{\prime}$, we consider that a fraction of the bubble energy $\left(E_{\mathrm{b}}^{\prime}=\eta M_{\mathrm{b}} c^{2}\right)$ is radiated at a constant rate $t_{\text {loss }}^{\prime-1}(z)$. The bubble either emits all its available energy, or escapes the jet in a time $t_{\mathrm{esc}} \sim z_{0} / c$. This results in long-lasting emission, generally dominated by one single event. If we consider emission through Inverse Compton processes, then $t_{\mathrm{acc}}^{\prime} \ll t_{\mathrm{IC}}^{\prime}$ for all considered situations, which results in luminosities of $\sim 10^{40} \mathrm{erg} s^{-1}$, for events (i.e. massive bubbles penetrating the jet) that occur a few times per century and last some $\sim 30$ yr. For low magnetic fields, synchrotron emission behaves similarly, as the characteristic time of losses is large enough. Through this emission mechanism, luminosities of $\sim 10^{42} \mathrm{erg}^{-1}$ can be reached, for events that take place a few times per century and last some $\sim 60 \mathrm{yr}$.

If the acceleration time is larger than the characteristic time of of energy losses, $t_{\text {acc }} \gg t_{\text {loss }}$, the energy is radiated rapidly and modulated by the efficiency of acceleration, as described in [26]. This results in flare-like emission, and occurs in the presence of very strong magnetic fields, when synchrotron emission is very intense. The total radiated energy in the synchrotron fast-cooling regime is [25]:

$$
E_{\mathrm{b}}^{\mathrm{app}}\left(M_{\mathrm{b}}, z\right)=\eta F_{\mathrm{rad}} \bar{F}_{\mathrm{e}} M_{\mathrm{b}} c^{2} \delta_{j}^{3}
$$

where we take $\bar{F}_{\mathrm{e}}=0.2$ [10], and $F_{\text {rad }}$ is the efficiency with which the particle loses energy through synchrotron radiation. In this scenario, which takes place for strong magnetic fields, we can reach much larger luminosities of $\sim 10^{43} \mathrm{erg} \mathrm{s}^{-1}$, for events that take place a few times per century and last only about $\sim 0.5 \mathrm{yr}$.

\section{Summary and Discussion}

We have described the possibility of stars interacting with the jet through stellar-wind bubbles at the moment of penetration, and given preliminary results for a particular case of red giants within an elliptical bulge.

According to our numerical estimations, this sort of interaction is unlikely to result in a dynamical impact on the jet, as the amount of mass loaded into it is insignificant when compared to the jet mass-rate. The same population of starts would also load into the jet about a factor $\sim 10^{4}$ more mass through their stellar winds during their jet-crossing time.

Our first estimates also suggest that, for the considered jet properties, it is possible to detect the emission generated in this interaction for nearby sources. Depending on the intensity of the 
magnetic field, it is possible that the synchrotron emission would be flare-like: bright and infrequent; or steady: dimmer and long-lasting, with duty cycles $\sim 1$. In the case of inverse Compton emission, we expect steady emission in all cases.

A more detailed study of this phenomenon, including sampling of different jet properties, is presented in [27].

\section{Acknowledgements}

We acknowledge support by the Spanish Ministerio de Economía y Competitividad (MINECO/ FEDER, UE) under grants AYA2013-47447-C3-1-P, AYA2016-76012-C3-1-P, with partial support by the European Regional Development Fund (ERDF/FEDER), MDM-2014-0369 of ICCUB (Unidad de Excelencia 'María de Maeztu'), and the Catalan DEC grant 2014 SGR 86. N.T-A. acknowledges support from MINECO through FPU14/04887 grant. V.B-R. also acknowledges financial support from MINECO and European Social Funds through a Ramón y Cajal fellowship. This research has been supported by the Marie Curie Career Integration Grant 321520.

\section{References}

[1] M. C. Begelman, R. D. Blandford and M. J. Rees, Theory of extragalactic radio sources, Reviews of Modern Physics 56 (1984) 255.

[2] I. Contopoulos, D. Gabuzda and N. Kylafis, eds., The Formation and Disruption of Black Hole Jets, vol. 414 of Astrophysics and Space Science Library, 2015. 10.1007/978-3-319-10356-3.

[3] V. S. Beskin, MHD Flows in Compact Astrophysical Objects: Accretion, Winds and Jets. Springer Verlag Berlin Heidelberg, 2009, 10.1007/978-3-642-01290-7.

[4] M. Boettcher, D. E. Harris and H. Krawczynski, Relativistic Jets from Active Galactic Nuclei. Wiley VCH Verlag GmbH \& Co. KGaA, Jan., 2012, 10.1002/9783527641741.

[5] A. Dar and A. Laor, Hadronic Production of TeV Gamma-Ray Flares from Blazars, 478 (1997) L5 [astro-ph/9610252].

[6] J. H. Beall and W. Bednarek, On the Hadronic Beam Model for Gamma-Ray Production in Blazars, 510 (1999) 188 [astro-ph/9802001].

[7] A. T. Araudo, V. Bosch-Ramon and G. E. Romero, Gamma rays from cloud penetration at the base of AGN jets, 522 (2010) A97 [1007.2199].

[8] W. Steffen, J. L. Gómez, A. C. Raga and R. J. R. Williams, Jet-Cloud Interactions and the Brightening of the Narrow-Line Region in Seyfert Galaxies, 491 (1997) L73 [astro-ph/9710178].

[9] W. Bednarek and R. J. Protheroe, Gamma-rays from interactions of stars with active galactic nucleus jets, 287 (1997) L9 [astro-ph/9612073].

[10] M. V. Barkov, F. A. Aharonian and V. Bosch-Ramon, Gamma-ray Flares from Red Giant/Jet Interactions in Active Galactic Nuclei, 724 (2010) 1517 [1 005 . 5252].

[11] A. T. Araudo, V. Bosch-Ramon and G. E. Romero, Gamma-ray emission from massive stars interacting with active galactic nuclei jets, 436 (2013) 3626 [1309.7114].

[12] M. Perucho, J. M. Martí, R. A. Laing and P. E. Hardee, On the deceleration of Fanaroff-Riley Class I jets: mass loading by stellar winds, 441 (2014) 1488 [1404.1209]. 
[13] V. Bosch-Ramon, Non-thermal emission from standing relativistic shocks: an application to red giant winds interacting with AGN jets, 575 (2015) A109 [1501.03118].

[14] V. M. de la Cita, V. Bosch-Ramon, X. Paredes-Fortuny, D. Khangulyan and M. Perucho, Coupling hydrodynamics and radiation calculations for star-jet interactions in active galactic nuclei, 591 (2016) A15 [1604.02070].

[15] S. Wykes, M. J. Hardcastle, A. I. Karakas and J. S. Vink, Internal entrainment and the origin of jet-related broad-band emission in Centaurus A, 447 (2015) 1001 [1409.5785].

[16] F. L. Vieyro, N. Torres-Albà and V. Bosch-Ramon, Collective non-thermal emission from an extragalactic jet interacting with stars, 604 (2017) A57 [1704.01919].

[17] W. Bednarek and P. Banasiński, Non-thermal Radiation from Collisions of Compact Objects with Intermediate-scale Jets in Active Galaxies, 807 (2015) 168 [1506.01181].

[18] B. L. Fanaroff and J. M. Riley, The morphology of extragalactic radio sources of high and low luminosity, 167 (1974) 31P.

[19] D. S. De Young, Mass entrainment in astrophysical jets, 307 (1986) 62.

[20] G. V. Bicknell, On the relationship between BL Lacertae objects and Fanaroff-Riley I radio galaxies, 422 (1994) 542 [astro-ph/9308033].

[21] Y. Wang, C. R. Kaiser, R. Laing, P. Alexander, G. Pavlovski and C. Knigge, A relativistic mixing-layer model for jets in low-luminosity radio galaxies, 397 (2009) 1113 [0905.2507].

[22] M. Perucho and J. M. Martí, A numerical simulation of the evolution and fate of a Fanaroff-Riley type I jet. The case of 3C 31, 382 (2007) 526 [0709.1784].

[23] S. S. Komissarov, Mass-Loaded Relativistic Jets, 269 (1994) 394.

[24] M. Bowman, J. P. Leahy and S. S. Komissarov, The deceleration of relativistic jets by entrainment, 279 (1996) 899.

[25] D. V. Khangulyan, M. V. Barkov, V. Bosch-Ramon, F. A. Aharonian and A. V. Dorodnitsyn, Star-Jet Interactions and Gamma-Ray Outbursts from 3C454.3, 774 (2013) 113 [1305. 5117].

[26] M. V. Barkov, F. A. Aharonian, S. V. Bogovalov, S. R. Kelner and D. Khangulyan, Rapid TeV Variability in Blazars as a Result of Jet-Star Interaction, 749 (2012) 119 [1012 . 1787].

[27] N. Torres-Albà and V. Bosch-Ramon, Gamma rays from red giant wind bubbles entering the jets of elliptical host blazars, submitted for publication in A \& A (2018) .

\section{DISCUSSION}

J. BEALL QUESTION: Can you say where you put the population of red giants? The BLR, NLR or further out?

N. TORRES-ALBÀ ANSWER: We consider a population of red giants extending from a height of $1 \mathrm{pc}$ up to $3 \mathrm{kpc}$, which is the size of the bugle of M87. However, the majority of significant intercations occur at a height of $\sim 1 \mathrm{kpc}$. 
D. V. BISIKALO QUESTION: What is the luminosity of the bow-shock in front of the star crossing the jet? Did you compare it with the luminosity of the blob?

N. TORRES-ALBÀ ANSWER: The bow-shock does not radiate thermal emission efficiently at any point of the bubble evolution. In the non-relativistic regime, the transfer of energy from the jet to the bubble is inefficient; and in the relativistic regime the bubble is much too diluted for thermal emission to be significant.

C. MUÑOZ-TUÑóN: You propose that red giants stars can leave their wind behind and this eventually produce gamma-ray emission. How probable are these fast giant stars and how many of them are to cross the jet in a "standard" galaxy halo?

N. TORRES-ALBÀ ANSWER: We consider only red giants in the galaxy bulge, where the jet is still collimated. The population of red giants is large, with as many as $\sim 10^{9}$ red giants within the bulge, $\sim 10^{6}$ of them within the jet at all times. Moving with characteristic speeds of $\sim 350 \mathrm{~km}$ $\mathrm{s}^{-1}$, events are frequent. However, most events do not result in significant emission; and the ones that do have a frequency of about a few per century. 